\title{
Physical chronic conditions, multimorbidity and sedentary behavior amongst middle- aged and older adults in six low- and middle-income countries
}

\author{
Davy Vancampfort ${ }^{1,2^{*}}$ (D) Brendon Stubbs ${ }^{3,4,5}$ and Ai Koyanagi ${ }^{6,7}$
}

\begin{abstract}
Background: Sedentary behavior (SB) is, irrespective of a person's physical activity levels, associated with a wide range of deleterious outcomes such as diabetes, stroke and associated premature mortality. There are no nationally representative, multi-national, population-based studies investigating the relationship between SB, chronic conditions, and physical multimorbidity (i.e., two or more chronic physical conditions). Thus, this cross-sectional study aimed to assess the association between chronic conditions, physical multimorbidity and SB among community-dwelling adults in six low- and middle-income countries (LMICs). We also explored the influential factors of these relationships.

Method: The Study on Global Ageing and Adult Health (SAGE) survey included 34,129 adults aged $\geq 50$ years. SB was self-reported and expressed as a categorical variable [ $<8$ or $\geq 8 \mathrm{~h}$ per day (high SB)]. Eleven chronic physical conditions (angina, arthritis, asthma, chronic back pain, chronic lung disease, diabetes, edentulism, hearing problems, hypertension, stroke, visual impairment) were assessed. Multivariable logistic regression and mediation analyses were conducted.

Results: The prevalence of physical multimorbidity and high SB ( $\geq 8 \mathrm{~h} /$ day) were $45.5 \%$ (43.7\%-47.4\%) and 10.8\% $(9.7 \%-12.1 \%)$, respectively. The prevalence of high SB increased in a linear fashion from $7.1 \%$ in people with no chronic condition to $24.1 \%$ in those with $\geq 4$ chronic conditions. In the multivariable analysis, visual impairment $(O R=2.62)$, stroke $(O R=2.02)$, chronic back pain $(O R=1.70)$ hearing problems $(O R=1.58)$, chronic lung disease $(O R=1.48)$, asthma $(O R=1.39)$, arthritis $(O R=1.22)$ and multimorbidity $(O R=1.41)$ were significantly associated with high SB. Disability explained more than $50 \%$ of the association for all chronic conditions with particularly high percentages (>80\%) for arthritis, asthma, and multimorbdity. Mobility problems explained $88.1 \%$ and $85.1 \%$ of the association of SB with arthritis and physical multimorbidiy, respectively. Pain was highly influential in the SB-arthritis relationship (85.6\%). Sleep/energy problems explained between 9.3\% (stroke) to 49.1\% (arthritis) of the association, and cognitive problems from 21.5\% (stroke) to 33.4\% (hearing problems). Findings for anxiety and depression were mixed.
\end{abstract}

Conclusion: In LMICS, those with chronic conditions and physical multimorbidity are significantly more sedentary. Targeted messages to reduce time spent sedentary among individuals with chronic conditions may ameliorate associated disability, mobility difficulties and pain that are themselves the most important risk factors for SB.

Keywords: Multimorbidity, Pain, Mobility limitation, Sitting, Sedentary time

\footnotetext{
* Correspondence: davy.vancampfort@kuleuven.be

${ }^{1} \mathrm{KU}$ Leuven Department of Rehabilitation Sciences, Tervuursevest 101, 3001

Leuven, Belgium

${ }^{2}$ KU Leuven, University Psychiatric Center KU Leuven, Leuvensesteenweg 517,

3070 Kortenberg, Belgium

Full list of author information is available at the end of the article
} 


\section{Background}

The average life expectancy is increasing worldwide [1]. Irrespective of the socioeconomic development of a country, the main causes of death and disability in middle-aged to older adults (i.e. 50 years or older) are non-communicable diseases (NCDs), many of which often occur in combination [2]. The co-occurrence of two or more chronic physical conditions is known as physical multimorbidity [3]. With increasing population and life expectancy, the disease burden of physical multimorbidity to both individuals and societies are increasing [4]. Although data are scarce, in low- and middle-income countries (LMICs) about half of middle-aged to older adults experience physical multimorbidity (i.e. 2 or more chronic conditions), about $25 \%$ have at least three, and about $10 \%$ four or more chronic conditions [5]. Physical multimorbidity is an important risk concept as it is associated with a lower quality of life [6], increased health-care utilization and costs [7], and ultimately, higher risk for premature mortality [8]. There is a consensus that in the years to come, this disease burden will be greatest in LMICs [9], but knowledge on physical multimorbidity from LMICs is limited compared with HICs (95\% of the available studies are from HICs), despite the fact that nearly $80 \%$ of NCD related deaths occur in LMICs [10].

The promotion of physical activity is an essential strategy within the multifaceted care of middle-aged to older adults for the prevention and management of chronic disease and physical multimorbidity [11]. Regular physical activity contributes to the primary and secondary prevention of a wide range of chronic diseases [12], improves quality of life [13], and is associated with reduced risk of premature death [14]. There is also increasing evidence demonstrating that sedentary behavior (SB) should be considered in this multifaceted care as it is, irrespective of a person's physical activity levels, associated with a wide range of deleterious outcomes such as diabetes, stroke and associated premature mortality [15]. $\mathrm{SB}$ refers to any waking behavior characterized by an energy expenditure $\leq 1.5$ metabolic equivalents (METs), while in a sitting, reclining or lying posture [16]. SB is highly prevalent and pervasive in societies across the world [17, 18]. A recent systematic review, virtually exclusively among western societies, demonstrated that middle-aged to older adults spend $65-80 \%$ of their waking day being sedentary [19].

Research investigating the relationship between SB and chronic diseases has solely focused on high-income countries. In addition, there is a particular dearth of research on SB and physical multimorbidity. In the only study on this topic to date, among 2048 American adults (mean age $=42.7$ years; $50.9 \%$ male), every $60 \mathrm{~min} /$ day increase in SB was associated with an 11\% (adjusted OR $=1.11 ; 95 \% \mathrm{CI}=1.01-1.21 ; P=0.03)$ increased odds of being multimorbid (i.e. having $\geq 2$ morbidities) [20]. Moreover, the authors found that SB was associated with multimorbidity independent of light-intensity physical activity and adherence to moderate-to-vigorous physical activity guidelines, which underscores the importance of minimizing prolonged SB (in addition to promoting physical activity) in the treatment of multimorbidity.

The lack of nationally representative population-based studies investigating the associations between $\mathrm{SB}$, chronic conditions, and physical multimorbidity in LMICs is an important research gap given the rapid increase in chronic diseases in these countries, mainly due to changes in lifestyle [21]. Furthermore, the association between chronic conditions or multimorbidity on SB may differ in LMICs due to different disease profiles [22], suboptimal treatment of chronic conditions [23, 24], and differences in knowledge regarding the risks of being sedentary [25]. In addition, at the population level, there is a paucity of information on factors that might influence the relationship between SB, chronic diseases and physical multimorbidity. In this study we focused on physical (mobility, disability, pain and discomfort) and mental (cognition, sleep and energy, anxiety and depression) conditions as these variables have all been associated with SB [26, 27] and the presence of chronic conditions [5]. Given the aforementioned gaps within the literature, we aimed to assess the association between chronic conditions or physical multimorbidity and SB among community-dwelling adults aged 50 or older in six LMICs, and to assess the factors that might influence this relationship. We hypothesize that higher levels of SB are associated with the presence of chronic conditions and physical multimorbidity.

\section{Methods}

\section{The survey}

Data from the SAGE were analyzed. This survey was undertaken in China, Ghana, India, Mexico, Russia, and South Africa between 2007 and 2010. Based on the World Bank classification at the time of the survey, all of these countries were LMICs. Details of the survey methodology have been published elsewhere [28]. In brief, in order to obtain nationally representative samples, a multistage clustered sampling design method was used. The sample consisted of adults aged $\geq 18$ years with oversampling of those aged $\geq 50$ years. Trained interviewers conducted face-to-face interviews using a standard questionnaire. Standard translation procedures were undertaken to ensure comparability between countries. If a respondent was unable to undertake the interview because of limited cognitive function, then a separate questionnaire was administered to a proxy respondent. These individuals were not included in the current study. The survey response rate ranged from 51\% (Mexico) to 93\% (China). Sampling weights were 
constructed to adjust for the population structure as reported by the United Nations Statistical Division. Ethical approval was obtained from the WHO Ethical Review Committee and local ethics research review boards. Written informed consent was obtained from all participants.

\section{Sedentary behaviour (outcome variable)}

In order to assess SB, participants were asked to state the total time they usually spent (expressed in minutes per day) sitting or reclining including at work, at home, getting to and from places, or with friends (e.g. sitting at a desk, sitting with friends, travelling in car, bus, train, reading, playing cards or watching television). This did not include time spent sleeping. The variable on SB was used in the analysis as a categorical $[<8$ (coded 0$)$ or $\geq 8 \mathrm{~h}$ (coded 1) per day] variable. The eight-hour cut-off was chosen as previous research indicated that being sedentary for $\geq 8 \mathrm{~h} /$ day in the general population is associated with a higher risk for premature mortality [29].

\section{Chronic conditions and physical multimorbidity (exposures)} Eleven chronic physical conditions (angina, arthritis, asthma, chronic back pain, chronic lung disease, diabetes, edentulism, hearing problems, hypertension, stroke, visual impairment) were assessed. Chronic back pain was defined as having had back pain everyday during the last 30 days. Respondents who answered affirmatively to the question "Have you lost all of your natural teeth?" were considered to have edentulism. The participant was considered to have hearing problems if the interviewer observed this condition during the survey. Blood pressure was measured three times with a one-minute interval with the use of a wrist blood pressure monitor (Medistar Wrist Blood Pressure Model S) and the mean value of the three measurements was calculated. Hypertension was defined as having at least one of the following: systolic blood pressure $\geq 140 \mathrm{mmHg}$; diastolic blood pressure $\geq 90 \mathrm{mmHg}$; or self-reported diagnosis. Visual impairment was defined as having extreme difficulty in seeing and recognizing a person that the participant knows across the road [30]. A validity study showed that this response likely corresponds to WHO definitions of visual impairment (20/60 or 0.48 $\operatorname{logMAR})[30]$. Diabetes and stroke were solely based on lifetime self-reported diagnosis.

For other conditions, the participant was considered to have the condition in the presence of either one of the following: self-reported diagnosis; or symptom-based diagnosis based on algorithms. We used these algorithms, which have been used in previous studies using the same dataset, to detect undiagnosed cases [31, 32]. Specifically, the validated Rose questionnaire was used for angina [33], and other previously validated symptombased algorithms were used for arthritis, asthma, and chronic lung disease [31, 34]. The questions used to assess self-reported diagnosis and the symptom-based algorithms are provided in Additional file 1: Tables S1 and S2. The total number of chronic conditions was calculated and categorized as $0,1,2,3$, and $\geq 4$. Multimorbidity was defined as $\geq 2$ chronic conditions [32].

\section{Mediators \\ Health status}

Health status was evaluated with eight health-related questions pertaining to four health domains including: (a) mobility; (b) pain and discomfort; (c) cognition; and (d) sleep and energy. Each of the four domains corresponds to those in common health related quality of life outcome measures such as the Short Form-12 (SF-12) [35], the Health Utilities Index Mark-3 (HUI) [36] and the EUROQOL-5D [37]. Each domain consists of two questions that assessed health function in the past 30 days. The actual questions can be found in Additional file 1: Table S3. Each item was scored on a five-point scale ranging from 'none' to 'extreme/cannot do'. For each separate domain, we used factor analysis with polychoric correlations to obtain a factor score which was later converted to scores ranging from 0 to 100 with higher values representing worse health function $[38,39]$.

\section{Anxiety}

In accordance with previous publications using a dataset with the identical question, those who claimed to have severe/extreme problems with worry or anxiety in the past 30 days were considered to have anxiety [40, 41].

\section{Depression}

Questions based on the World Mental Health Survey version of the Composite International Diagnostic Interview [42] were used for the endorsement of past 12month DSM-IV depression using the same algorithm used in previous studies using the same dataset [32, 43] (Details provided in Additional file 1: Table S4).

\section{Disability}

Disability was assessed by the use of the 12-item validated version of the World Health Organization Disability Assessment Schedule 2.0 (WHODAS 2.0) [44]. Item Response Theory analysis was used to create a scale ranging from 0 (no disability) to 100 (maximum disability) [45].

\section{Control variables}

These included sex, age, wealth, highest level of education achieved ( $\leq$ primary, secondary, $\geq$ tertiary), setting (urban or rural), living arrangement (alone or not), and employment status (engaged in paid work $\geq 2$ days in last 
7 days: $\mathrm{Y} / \mathrm{N})$. Wealth quintiles were created based on country-specific income.

\section{Statistical analysis}

The statistical analysis was performed with Stata 14.1 (Stata Corp LP, College station, Texas). The analysis was restricted to those aged $\geq 50$ years given the high prevalence of chronic physical conditions in this age group. We conducted multivariable logistic regression analysis to assess the association between the number of chronic conditions including physical multimorbidity ( $\geq 2$ chronic physical conditions) or each of the 11 chronic conditions (exposure variables) and sedentary behavior (outcome). Analyses using the overall sample and by age groups (50-64, $\geq 65$ years) was done. Next, in order to gain an understanding of the extent to which various factors may explain the relation of individual chronic conditions and physical multimorbidity with $\mathrm{SB}$, we conducted mediation analysis using the overall sample. We did not conduct this analysis for angina, diabetes, edentulism, and hypertension as these conditions were not significantly associated with SB in the overall sample. We focused on anxiety, cognition, depression, disability, mobility, pain/discomfort, and sleep/energy for their previously reported association with the exposure (chronic physical conditions) and the outcome (sedentary behavior). We used the khb (Karlson Holm Breen) command in Stata [46] for this purpose. This method can be applied in logistic regression models and decomposes the total effect (i.e., unadjusted for the influential factor) of a variable into direct (i.e. the effect of chronic conditions or multimorbidity on SB adjusted for the factor) and indirect effects (i.e. the mediational effect). Using this method, the percentage of the main association explained by the mediator can also be calculated. Each potential mediator was included in the model individually. All regression analyses were adjusted for sex, age, education, wealth, setting, unemployment, living arrangement, and country. Country adjustment was done by including dummy variables for each country. When the individual chronic conditions were the exposure variable, the models were also adjusted for the presence of other chronic illness to account for comorbid chronic conditions. This variable included information on whether the individual had any of the other ten chronic conditions $(\mathrm{Y} / \mathrm{N})$. All variables were included in the models as categorical variables with the exception of age, pain/discomfort, cognition, sleep/energy, and disability (continuous variables). Under $5 \%$ of the data were missing for the variables used in the analysis. Complete-case analysis was done. The sample weighting and the complex study design were taken into account in the analyses. Results from the regression analyses are presented as odds ratios (ORs) with 95\% confidence intervals (CIs). The level of statistical significance was set at $P<0.05$.

\section{Results}

The final analytical sample consisted of 34,129 individuals (China $n=13,175$; Ghana $n=4305$; India $n=6560$; Mexico $n=2313$; Russia $n=3938$; South Africa $n=3838$ ) aged $\geq 50$ years. The median (IQR) age was $62(55-70)$ years and $47.9 \%$ were males. The prevalence of physical multimorbidity and high SB (i.e. $\geq 8 \mathrm{~h} /$ day) were $45.5 \%(43.7 \%-47.4 \%)$ and $10.8 \%(9.7 \%-12.1 \%)$, respectively (Table 1 ).

The prevalence of high SB increased in a linear fashion with an increasing number of chronic conditions ranging from $7.1 \%$ in people with no chronic condition to $24.1 \%$ in those with 4 or more chronic conditions (Fig. 1).

The results of the multivariable logistic regression analysis assessing the association between chronic conditions or physical multimorbidity and high SB are presented in Table 2. In the overall sample, visual impairment $(\mathrm{OR}=2.62)$, stroke $(\mathrm{OR}=2.02)$, chronic back pain $(\mathrm{OR}=1.70)$ hearing problems $(\mathrm{OR}=1.58)$, chronic lung disease $(\mathrm{OR}=1.48)$, asthma $(\mathrm{OR}=1.39)$, arthritis $(\mathrm{OR}=1.22)$, and physical multimorbidity $(\mathrm{OR}=1.41)$ were significantly associated with high SB. When the analysis was stratified by age groups, arthritis, chronic back pain, hearing problems, and visual impairment, and physical multimorbidity were only significantly associated with high SB in the older age group, while asthma was only associated with high SB in the middle-aged group $(P<0.05)$. In terms of the number of chronic conditions, in the overall sample, compared to those who no chronic conditions, the presence of one or 2 chronic conditions was not significantly associated with high $\mathrm{SB}$ but the presence of $3(\mathrm{OR}=1.55 ; p<0.001)$ and 4 or more $(\mathrm{OR}=2.22 ; \mathrm{p}<0.001)$ was associated with high SB. When stratified by age groups, in those aged 65 years or older, having 2 chronic conditions was significantly associated with higher odds $(\mathrm{OR}=1.67)$ for high $\mathrm{SB}$, while this odds increased to 2.36 and 2.98 for the presence of 3 and 4 or more chronic conditions, respectively $(P<0.001)$.

The results of the mediation analysis among those aged $\geq 50$ years are shown in Tables 3 and 4 .

For the individual chronic conditions, anxiety did not explain the association of high SB with arthritis and stroke while for other conditions it explained from $6.0 \%$ (hearing problems) to $23.5 \%$ (visual impairment) of the association. Cognitive problems explained the relationship from $21.5 \%$ (stroke) to $33.4 \%$ (hearing problems). Depression was not an influential factor in the association of high SB with hearing problems and stroke, while it explained between 6.2\% (chronic back pain) to $13.2 \%$ (asthma) of the association for the other conditions. 
Table 1 Sample characteristics (overall and by highly sedentary behavior)

\begin{tabular}{|c|c|c|c|c|c|}
\hline \multirow[b]{2}{*}{ Characteristic } & \multirow[b]{2}{*}{ Category } & \multirow[b]{2}{*}{$\mathrm{N}$} & \multirow[b]{2}{*}{ Overall } & \multicolumn{2}{|c|}{ Highly sedentary behavior ${ }^{a}$} \\
\hline & & & & No & Yes \\
\hline \multirow[t]{5}{*}{ Number of chronic conditions } & 0 & 6624 & 22.2 & 23.2 & 14.6 \\
\hline & 1 & 11,437 & 32.3 & 33.3 & 24.1 \\
\hline & 2 & 7430 & 22.4 & 22.6 & 20.7 \\
\hline & 3 & 3880 & 12.2 & 11.7 & 16.4 \\
\hline & $\geq 4$ & 3214 & 10.9 & 9.2 & 24.1 \\
\hline \multicolumn{6}{|l|}{ Sociodemographics } \\
\hline Age (years) & Median (IQR) & & $62(55-70)$ & $61(55-70)$ & $67(58-75)$ \\
\hline Sex & Male & 15,666 & 47.9 & 48.4 & 45.1 \\
\hline \multirow[t]{3}{*}{ Education } & $\leq$ Primary & 21,264 & 57.4 & 58.8 & 47.7 \\
\hline & Secondary & 9943 & 35.2 & 34.1 & 41.8 \\
\hline & $\geq$ Tertiary & 2189 & 7.4 & 7.1 & 10.5 \\
\hline \multirow[t]{5}{*}{ Wealth } & Poorest & 6496 & 17.1 & 16.6 & 20.7 \\
\hline & Poorer & 6697 & 19.0 & 18.5 & 21.7 \\
\hline & Middle & 6648 & 19.5 & 19.6 & 19.1 \\
\hline & Richer & 7009 & 21.3 & 21.8 & 18.0 \\
\hline & Richest & 7147 & 23.1 & 23.5 & 20.5 \\
\hline Setting & Urban & 17,115 & 46.2 & 45.4 & 51.5 \\
\hline Unemployed & Yes & 20,134 & 57.3 & 55.7 & 70.9 \\
\hline Living arrangement & Alone & 3608 & 10.0 & 8.8 & 18.8 \\
\hline \multicolumn{6}{|l|}{ Chronic physical conditions } \\
\hline Angina & Yes & 4921 & 17.6 & 16.4 & 25.5 \\
\hline Arthritis & Yes & 9514 & 29.5 & 28.5 & 37.3 \\
\hline Asthma & Yes & 2390 & 7.9 & 7.5 & 10.7 \\
\hline Chronic back pain & Yes & 2543 & 8.6 & 7.7 & 15.9 \\
\hline Chronic lung disease & Yes & 4477 & 15.8 & 14.5 & 25.2 \\
\hline Diabetes & Yes & 2647 & 6.8 & 6.7 & 8.4 \\
\hline Edentulism & Yes & 4124 & 12.9 & 12.1 & 19.7 \\
\hline Hearing problems & Yes & 2075 & 5.6 & 4.9 & 11.1 \\
\hline Hypertension & Yes & 20,141 & 55.0 & 54.0 & 61.9 \\
\hline Stroke & Yes & 1190 & 3.0 & 2.6 & 7.0 \\
\hline Visual impairment & Yes & 353 & 1.3 & 1.0 & 3.3 \\
\hline \multicolumn{6}{|l|}{ Health status } \\
\hline Mobility & Median (IQR) & & $27(0-58)$ & $27(0-51)$ & $51(27-73)$ \\
\hline Pain/discomfort & Median (IQR) & & $36(0-56)$ & $36(0-46)$ & $36(18-56)$ \\
\hline Cognition & Median (IQR) & & $50(40-69)$ & $31(0-50)$ & $41(20-60)$ \\
\hline Sleep/energy & Median (IQR) & & $20(0-49)$ & $20(0-40)$ & $40(0-59)$ \\
\hline \multicolumn{6}{|l|}{ Other variables } \\
\hline Disability & Median (IQR) & & $11(3-31)$ & $11(3-28)$ & $25(8-47)$ \\
\hline Anxiety & Yes & 2046 & 8.1 & 7.5 & 13.6 \\
\hline Depression & Yes & 1692 & 6.0 & 5.8 & 8.7 \\
\hline
\end{tabular}

Abbreviation: IQR Interquartile range

Data are column percentages unless otherwise stated

Estimates are based on weighted sample

${ }^{a}$ Highly sedentary behavior referred to being sedentary for $\geq 8 \mathrm{~h} /$ day 


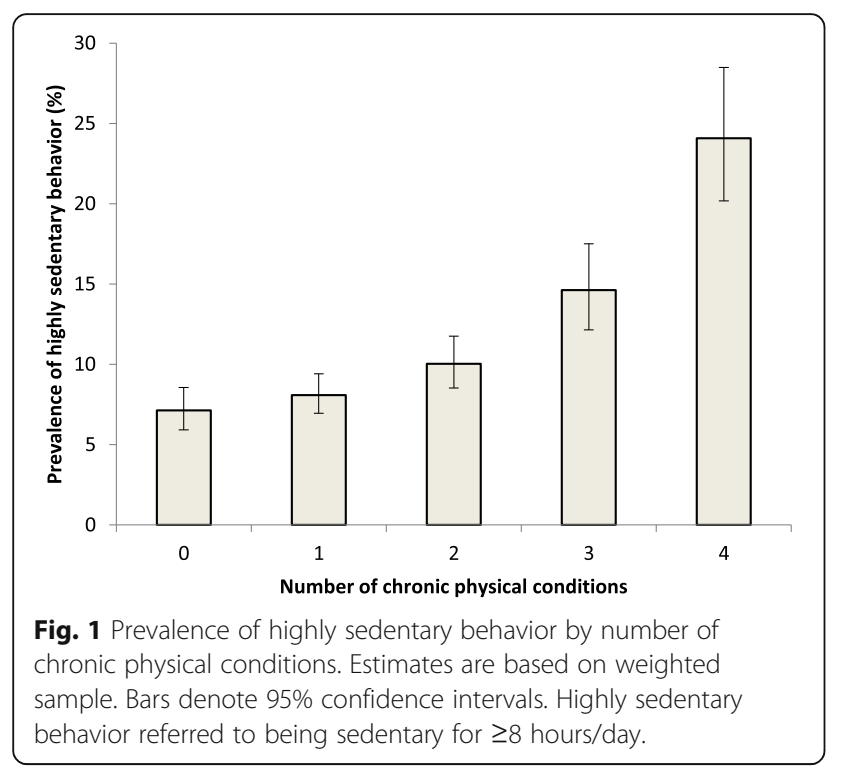

Sleep/energy problems explained 9.3\% (stroke) to $49.1 \%$ (arthritis) of the relationship. Disability explained more than $50 \%$ of the association for all chronic conditions with particularly high mediated percentages observed for arthritis (81.6\%) and asthma (80.6\%). Mobility difficulties also explained more than $35 \%$ of the association for all chronic conditions with particularly high mediated percentages observed for arthritis (88.1\%). Pain was a major influential factor for the association between SB and arthritis (85.6\%) and explained between 15.2\% (stroke) to $44.5 \%$ (asthma) of the association with other conditions.

As for the SB - physical multimorbidity relationship, the most important influential factor was disability (89.1\%), followed by mobility (85.1\%), pain/discomfort (60.2\%), sleep/energy (38.1\%), cognition (35.0\%), anxiety (11.5\%), and depression (7.5\%).

\section{Discussion}

\section{General findings}

To the best of our knowledge, the current study is the first large-scale $(n=34,129)$, multinational (6 LMICs) analysis investigating associations of $\mathrm{SB}$ with chronic conditions and physical multimorbidity. The current data confirm the findings from a previous study conducted in a high-income country showing that SB is associated with a higher risk for physical multimorbidity [20]. We found that most chronic conditions were associated with a high SB status (i.e. eight or more hours per day) in the overall sample, although this relationship was most notable among the oldest population (i.e. 65 years or older). Our mediation analysis showed that disability

Table 2 Associations of chronic conditions, multimorbidity, and number of chronic conditions with highly sedentary behavior (outcome) estimated by multivariable logistic regression

\begin{tabular}{lllll}
\hline & & Overall & $50-64$ years & $\geq 65$ years \\
\hline Angina & Yes vs. No & $1.04[0.83,1.31]$ & $1.00[0.71,1.41]$ & $1.10[0.84,1.45]$ \\
Arthritis & Yes vs. No & $1.22^{*}[1.03,1.44]$ & $1.17[0.92,1.49]$ & $1.33^{*}[1.07,1.67]$ \\
Asthma & Yes vs. No & $1.39^{*}[1.07,1.80]$ & $1.59^{*}[1.02,2.47]$ & $1.24[0.95,1.61]$ \\
Chronic back pain & Yes vs. No & $1.70^{* * *}[1.37,2.11]$ & $1.38[0.98,1.95]$ & $1.87^{* * *}[1.43,2.44]$ \\
Chronic lung disease & Yes vs. No & $1.48^{* * *}[1.19,1.85]$ & $1.69^{* * *}[1.26,2.26]$ & $1.38^{*}[1.05,1.82]$ \\
Diabetes & Yes vs. No & $1.15[0.93,1.44]$ & $1.24[0.89,1.72]$ & $1.15[0.88,1.49]$ \\
Edentulism & Yes vs. No & $1.11[0.86,1.43]$ & $0.87[0.61,1.26]$ & $1.14[0.84,1.54]$ \\
Hearing problems & Yes vs. No & $1.58^{* * *}[1.23,2.02]$ & $1.20[0.68,2.11]$ & $1.54^{* *}[1.16,2.03]$ \\
Hypertension & Yes vs. No & $0.98[0.85,1.14]$ & $0.98[0.79,1.22]$ & $1.03[0.85,1.26]$ \\
Stroke & Yes vs. No & $2.02^{* * *}[1.53,2.67]$ & $2.20^{* * *}[1.46,3.32]$ & $2.09^{* * *}[1.46,3.00]$ \\
Visual impairment & Yes vs. No & $2.62^{* * *}[1.75,3.93]$ & $2.11[0.86,5.19]$ & $2.45^{* * *}[1.52,3.95]$ \\
Multimorbidity ${ }^{2}$ & Yes vs. No & $1.41^{* * *}[1.19,1.66]$ & $1.17[0.93,1.48]$ & $1.81^{* * *}[1.39,2.36]$ \\
Number of chronic conditions & 0 & 1.00 & 1.00 & 1.00 \\
& 1 & $1.09[0.88,1.37]$ & $1.07[0.82,1.40]$ & $1.35[0.97,1.88]$ \\
& 2 & $1.16[0.93,1.46]$ & $1.01[0.75,1.36]$ & $1.10[0.69,1.74]$ \\
& 3 & $1.55^{* * *}[1.21,1.98]$ & $2.26^{* * *}[1.44,3.53]$ & $2.36^{* * * *}[1.58,3.52]$ \\
& $\geq 4$ & $2.22^{* * *}[1.68,2.94]$ & & $2.98^{* * *}[1.96,4.51]$ \\
\hline
\end{tabular}

Data are odds ratio [95\% confidence interval]

Highly sedentary behavior referred to being sedentary for $\geq 8 \mathrm{~h} /$ day

Models are adjusted for sex, age, education, wealth, setting, unemployment, living arrangement, and country. For individual chronic conditions, the models were also adjusted for the presence of other chronic conditions

${ }^{*} p<0.05$, ** $p<0.01,{ }^{* * *} p<0.001$

${ }^{\text {a }}$ Multimorbidity was defined as $\geq 2$ chronic conditions 
Table 3 Mental health (anxiety, cognition, depression, sleep/energy) variables as mediators in the association between chronic conditions (multimorbidity) and highly sedentary behavior among adults aged $\geq 50$ years

\begin{tabular}{|c|c|c|c|c|c|c|c|c|}
\hline \multirow[b]{2}{*}{ Exposure } & \multirow[b]{2}{*}{ Mediator } & \multicolumn{2}{|l|}{ Total effect } & \multicolumn{2}{|l|}{ Direct effect } & \multicolumn{2}{|l|}{ Indirect effect } & \multirow[b]{2}{*}{$\%$ Mediated } \\
\hline & & OR $[95 \% \mathrm{Cl}]$ & $P$-value & OR [95\% Cl] & $P$-value & OR $[95 \% \mathrm{Cl}]$ & $P$-value & \\
\hline \multirow[t]{4}{*}{ Arthritis } & Anxiety & $1.22[1.04,1.45]$ & 0.017 & $1.21[1.03,1.43]$ & 0.023 & $1.01[1.00,1.02]$ & 0.064 & NA \\
\hline & Cognition & $1.23[1.04,1.46]$ & 0.014 & $1.15[0.97,1.37]$ & 0.108 & $1.07[1.04,1.10]$ & $<0.001$ & 32.8 \\
\hline & Depression & $1.22[1.04,1.45]$ & 0.018 & $1.21[1.02,1.43]$ & 0.030 & $1.02[1.01,1.03]$ & 0.003 & 7.7 \\
\hline & Sleep/energy & $1.23[1.04,1.45]$ & 0.017 & $1.11[0.94,1.31]$ & 0.225 & $1.11[1.07,1.14]$ & $<0.001$ & 49.1 \\
\hline \multirow[t]{4}{*}{ Asthma } & Anxiety & $1.38[1.06,1.81]$ & 0.017 & $1.30[0.99,1.70]$ & 0.059 & $1.06[1.03,1.10]$ & $<0.001$ & 19.3 \\
\hline & Cognition & $1.40[1.07,1.83]$ & 0.014 & $1.27[0.97,1.66]$ & 0.082 & $1.10[1.06,1.14]$ & $<0.001$ & 28.7 \\
\hline & Depression & $1.39[1.06,1.81]$ & 0.016 & $1.33[1.02,1.74]$ & 0.036 & $1.04[1.02,1.07]$ & 0.003 & 13.2 \\
\hline & Sleep/energy & $1.38[1.07,1.79]$ & 0.014 & $1.21[0.93,1.56]$ & 0.155 & $1.15[1.10,1.20]$ & $<0.001$ & 42.3 \\
\hline \multirow[t]{4}{*}{ Chronic back pain } & Anxiety & $1.71[1.36,2.14]$ & $<0.001$ & $1.61[1.28,2.03]$ & $<0.001$ & $1.06[1.03,1.09]$ & $<0.001$ & 10.6 \\
\hline & Cognition & $1.74[1.38,2.18]$ & $<0.001$ & $1.48[1.18,1.86]$ & 0.001 & $1.17[1.12,1.23]$ & $<0.001$ & 28.7 \\
\hline & Depression & $1.71[1.37,2.14]$ & $<0.001$ & $1.65[1.32,2.07]$ & $<0.001$ & $1.03[1.01,1.06]$ & 0.003 & 6.2 \\
\hline & Sleep/energy & $1.70[1.36,2.13]$ & $<0.001$ & $1.50[1.19,1.89]$ & 0.001 & $1.13[1.08,1.18]$ & $<0.001$ & 23.6 \\
\hline \multirow[t]{4}{*}{ Chronic lung disease } & Anxiety & $1.48[1.18,1.85]$ & 0.001 & $1.39[1.11,1.74]$ & 0.005 & $1.07[1.04,1.10]$ & $<0.001$ & 16.4 \\
\hline & Cognition & $1.48[1.19,1.84]$ & $<0.001$ & $1.33[1.07,1.66]$ & 0.011 & $1.11[1.08,1.15]$ & $<0.001$ & 27.6 \\
\hline & Depression & $1.48[1.19,1.85]$ & 0.001 & $1.43[1.15,1.79]$ & 0.001 & $1.03[1.01,1.05]$ & 0.002 & 8.2 \\
\hline & Sleep/energy & $1.49[1.19,1.85]$ & $<0.001$ & $1.32[1.06,1.65]$ & 0.013 & $1.13[1.08,1.17]$ & $<0.001$ & 29.9 \\
\hline \multirow[t]{4}{*}{ Hearing problems } & Anxiety & $1.58[1.24,2.03]$ & $<0.001$ & $1.54[1.21,1.97]$ & 0.001 & $1.03[1.00,1.05]$ & 0.036 & 6.0 \\
\hline & Cognition & $1.60[1.24,2.05]$ & $<0.001$ & $1.36[1.06,1.75]$ & 0.015 & $1.17[1.12,1.22]$ & $<0.001$ & 33.4 \\
\hline & Depression & $1.57[1.22,2.02]$ & 0.001 & $1.57[1.21,2.02]$ & 0.001 & $1.00[0.99,1.02]$ & 0.842 & NA \\
\hline & Sleep/energy & $1.59[1.24,2.05]$ & $<0.001$ & $1.50[1.17,1.94]$ & 0.002 & $1.06[1.03,1.09]$ & $<0.001$ & 12.2 \\
\hline \multirow[t]{4}{*}{ Stroke } & Anxiety & $2.02[1.52,2.69]$ & $<0.001$ & $1.97[1.48,2.61]$ & $<0.001$ & $1.03[1.00,1.06]$ & 0.054 & NA \\
\hline & Cognition & $2.03[1.56,2.66]$ & $<0.001$ & $1.75[1.33,2.29]$ & $<0.001$ & $1.16[1.11,1.23]$ & $<0.001$ & 21.5 \\
\hline & Depression & $2.03[1.54,2.66]$ & $<0.001$ & $2.00[1.52,2.63]$ & $<0.001$ & $1.01[1.00,1.03]$ & 0.112 & NA \\
\hline & Sleep/energy & $2.03[1.56,2.64]$ & $<0.001$ & $1.90[1.46,2.48]$ & $<0.001$ & $1.07[1.03,1.11]$ & $<0.001$ & 9.3 \\
\hline \multirow[t]{4}{*}{ Visual impairment } & Anxiety & $2.64[1.75,3.99]$ & $<0.001$ & $2.10[1.38,3.21]$ & 0.001 & $1.26[1.13,1.39]$ & $<0.001$ & 23.5 \\
\hline & Cognition & $2.66[1.73,4.10]$ & $<0.001$ & $1.97[1.27,3.06]$ & 0.002 & $1.35[1.23,1.48]$ & $<0.001$ & 30.7 \\
\hline & Depression & $2.60[1.73,3.91]$ & $<0.001$ & $2.43[1.62,3.65]$ & $<0.001$ & $1.07[1.02,1.12]$ & 0.009 & 6.9 \\
\hline & Sleep/energy & $2.64[1.75,3.99]$ & $<0.001$ & $2.29[1.51,3.47]$ & $<0.001$ & $1.15[1.08,1.23]$ & $<0.001$ & 14.6 \\
\hline \multirow[t]{4}{*}{ Multimorbidity $^{a}$} & Anxiety & $1.41[1.20,1.67]$ & $<0.001$ & $1.36[1.15,1.60]$ & $<0.001$ & $1.04[1.02,1.06]$ & $<0.001$ & 11.5 \\
\hline & Cognition & $1.40[1.19,1.65]$ & $<0.001$ & $1.24[1.05,1.47]$ & 0.012 & $1.12[1.09,1.16]$ & $<0.001$ & 35.0 \\
\hline & Depression & $1.41[1.19,1.66]$ & $<0.001$ & $1.37[1.16,1.62]$ & $<0.001$ & $1.03[1.01,1.04]$ & 0.001 & 7.5 \\
\hline & Sleep/energy & $1.40[1.19,1.66]$ & $<0.001$ & $1.23[1.05,1.45]$ & 0.012 & $1.14[1.09,1.19]$ & $<0.001$ & 38.1 \\
\hline
\end{tabular}

Abbreviation: OR Odds ratio, CI Confidence interval

Highly sedentary behavior referred to being sedentary for $\geq 8 \mathrm{~h} /$ day

Models are adjusted for sex, age, education, wealth, setting, unemployment, living arrangement, and country. For individual chronic conditions, the models were also adjusted for the presence of other chronic conditions

The mediated percentage was only calculated in the presence of a significant indirect effect $(P<0.05)$

${ }^{a}$ Multimorbidity was defined as $\geq 2$ chronic conditions

and mobility difficulties were important factors for most of the chronic conditions studied, while pain was a central factor for arthritis. As for physical multimorbidity, disability, mobility difficulties and pain were also important factors mediating high SB. Sleep/energy and cognitive problems explained the relation between $\mathrm{SB}$ and each chronic condition to a lesser extent while findings for anxiety, and depression were mixed. The factors identified in our study, which may explain the relationship between chronic conditions and physical multimorbidity with SB, should however be confirmed in prospective studies.

In the overall sample, those with arthritis $(\mathrm{OR}=1.22)$ and chronic back pain $(\mathrm{OR}=1.70)$ were more likely to 
Table 4 Disability, mobility, and pain/discomfort as mediators in the association between chronic conditions (multimorbidity) and highly sedentary behavior among adults aged $\geq 50$ years

\begin{tabular}{|c|c|c|c|c|c|c|c|c|}
\hline \multirow[b]{2}{*}{ Exposure } & \multirow[b]{2}{*}{ Mediator } & \multicolumn{2}{|l|}{ Total effect } & \multicolumn{2}{|l|}{ Direct effect } & \multicolumn{2}{|l|}{ Indirect effect } & \multirow[b]{2}{*}{$\%$ Mediated } \\
\hline & & $\mathrm{OR}[95 \% \mathrm{Cl}]$ & $P$-value & OR $[95 \% \mathrm{Cl}]$ & $P$-value & OR [95\% Cl] & $P$-value & \\
\hline \multirow[t]{3}{*}{ Arthritis } & Disability & $1.23[1.04,1.46]$ & 0.017 & $1.04[0.87,1.24]$ & 0.670 & $1.18[1.14,1.23]$ & $<0.001$ & 81.6 \\
\hline & Mobility & $1.23[1.04,1.45]$ & 0.015 & $1.02[0.86,1.22]$ & 0.780 & $1.20[1.15,1.25]$ & $<0.001$ & 88.1 \\
\hline & Pain/discomfort & $1.23[1.04,1.46]$ & 0.016 & $1.03[0.87,1.22]$ & 0.732 & $1.19[1.14,1.25]$ & $<0.001$ & 85.6 \\
\hline \multirow[t]{3}{*}{ Asthma } & Disability & $1.41[1.07,1.85]$ & 0.014 & $1.07[0.81,1.41]$ & 0.638 & $1.32[1.24,1.40]$ & $<0.001$ & 80.6 \\
\hline & Mobility & $1.41[1.08,1.84]$ & 0.011 & $1.12[0.86,1.45]$ & 0.413 & $1.27[1.20,1.33]$ & $<0.001$ & 68.4 \\
\hline & Pain/discomfort & $1.41[1.08,1.84]$ & 0.011 & $1.21[0.93,1.58]$ & 0.158 & $1.17[1.11,1.22]$ & $<0.001$ & 44.5 \\
\hline \multirow[t]{3}{*}{ Chronic back pain } & Disability & $1.71[1.35,2.17]$ & $<0.001$ & $1.28[1.02,1.60]$ & 0.035 & $1.34[1.26,1.43]$ & $<0.001$ & 54.7 \\
\hline & Mobility & $1.75[1.38,2.22]$ & $<0.001$ & $1.36[1.08,1.72]$ & 0.009 & $1.29[1.22,1.36]$ & $<0.001$ & 44.9 \\
\hline & Pain/discomfort & $1.73[1.37,2.17]$ & $<0.001$ & $1.36[1.09,1.69]$ & 0.007 & $1.27[1.19,1.36]$ & $<0.001$ & 43.8 \\
\hline \multirow[t]{3}{*}{ Chronic lung disease } & Disability & $1.49[1.19,1.86]$ & $<0.001$ & $1.16[0.93,1.45]$ & 0.191 & $1.28[1.22,1.35]$ & $<0.001$ & 62.5 \\
\hline & Mobility & $1.50[1.21,1.87]$ & $<0.001$ & $1.19[0.96,1.46]$ & 0.110 & $1.27[1.21,1.33]$ & $<0.001$ & 58.1 \\
\hline & Pain/discomfort & $1.49[1.20,1.85]$ & $<0.001$ & $1.27[1.02,1.58]$ & 0.032 & $1.18[1.12,1.23]$ & $<0.001$ & 40.4 \\
\hline \multirow[t]{3}{*}{ Hearing problems } & Disability & $1.58[1.21,2.05]$ & 0.001 & $1.19[0.91,1.55]$ & 0.194 & $1.32[1.24,1.42]$ & $<0.001$ & 61.4 \\
\hline & Mobility & $1.60[1.23,2.07]$ & $<0.001$ & $1.35[1.04,1.75]$ & 0.024 & $1.18[1.13,1.24]$ & $<0.001$ & 35.8 \\
\hline & Pain/discomfort & $1.59[1.23,2.05]$ & $<0.001$ & $1.46[1.13,1.87]$ & 0.003 & $1.09[1.05,1.13]$ & $<0.001$ & 18.6 \\
\hline \multirow[t]{3}{*}{ Stroke } & Disability & $2.01[1.52,2.67]$ & $<0.001$ & $1.40[1.04,1.87]$ & 0.026 & $1.44[1.32,1.58]$ & $<0.001$ & 52.3 \\
\hline & Mobility & $2.02[1.54,2.65]$ & $<0.001$ & $1.48[1.12,1.98]$ & 0.007 & $1.36[1.27,1.46]$ & $<0.001$ & 43.8 \\
\hline & Pain/discomfort & $2.04[1.57,2.66]$ & $<0.001$ & $1.83[1.41,2.39]$ & $<0.001$ & $1.11[1.07,1.17]$ & $<0.001$ & 15.2 \\
\hline \multirow[t]{3}{*}{ Visual impairment } & Disability & $2.79[1.85,4.22]$ & $<0.001$ & $1.35[0.88,2.05]$ & 0.165 & $2.08[1.77,2.43]$ & $<0.001$ & 71.1 \\
\hline & Mobility & $2.76[1.80,4.25]$ & $<0.001$ & $1.79[1.16,2.74]$ & 0.008 & $1.55[1.40,1.71]$ & $<0.001$ & 43.0 \\
\hline & Pain/discomfort & $2.68[1.77,4.06]$ & $<0.001$ & $2.09[1.38,3.17]$ & 0.001 & $1.28[1.19,1.39]$ & $<0.001$ & 25.4 \\
\hline \multirow[t]{3}{*}{ Multimorbidity $^{a}$} & Disability & $1.36[1.15,1.61]$ & $<0.001$ & $1.03[0.87,1.23]$ & 0.705 & $1.32[1.25,1.39]$ & $<0.001$ & 89.1 \\
\hline & Mobility & $1.39[1.18,1.64]$ & $<0.001$ & $1.05[0.89,1.24]$ & 0.570 & $1.32[1.26,1.40]$ & $<0.001$ & 85.1 \\
\hline & Pain/discomfort & $1.40[1.18,1.66]$ & $<0.001$ & $1.14[0.97,1.35]$ & 0.119 & $1.22[1.16,1.30]$ & $<0.001$ & 60.2 \\
\hline
\end{tabular}

Abbreviation: $O R$ Odds ratio, $\mathrm{Cl}$ Confidence interval

Highly sedentary behavior referred to being sedentary for $\geq 8 \mathrm{~h} /$ day

Models are adjusted for sex, age, education, wealth, setting, unemployment, living arrangement, and country. For individual chronic conditions, the models were also adjusted for the presence of other chronic conditions

The mediated percentage was only calculated in the presence of a significant indirect effect $(P<0.05)$

${ }^{a}$ Multimorbidity was defined as $\geq 2$ chronic conditions

be sedentary for eight or more hours per day. These relationships appear to be mainly explained by disability, mobility difficulties, and pain while cognitive problems, depressive feelings, and sleep problems may also play a role. In both conditions, pain might cause mobility and sleep problems and ultimately feelings of depression, which in turn can all result in people being more sedentary. Previous literature in Western populations has demonstrated that pain can result in mobility limitations, higher levels of SB [47], and depression [48]. Clearly, such an interplay of pain, mobility limitations and depression among people with physical multimorbidity could play an even more pronounced role. Next to this, some SBs such as TV viewing, have been linked previously to poorer cognitive function in middle-aged to older adults [49], although this is largely drawn from high-income populations. The reason why adults with cognitive problems are more likely to be sedentary is largely unknown, but clearly this warrants further exploration. One possible explanation is that cognitive problems, such as impairments in executive functioning can result in difficulties in planning complex behaviors and consequently there might be an increased risk of falls [50]. Falls are on its turn associated with a fear of falling again and avoidance of activities due to this fear, which is also associated with grey matter volume loss $[51,52]$. In the case of arthritis, cognitive problems may be linked to SB via comorbid cardiovascular risk factors or systemic inflammatory processes [53, 54]. Sedentary time and the number of sedentary bouts per day (i.e. $\geq 20 \mathrm{~min}$ ) are associated with an increased 10 year cardiovascular disease (CVD) risk in people with 
arthritis, independent of engagement in moderate to vigorous physical activity [55].

Our data also suggest that people with asthma were more sedentary $(\mathrm{OR}=1.39)$. It is known that many individuals with asthma avoid physical activities for fear of exacerbating symptoms or triggering physical activity-induced bronchoconstriction [56]. Studies have shown that replacing SB with light intensity physical activity can however improve asthma control and asthma-related quality of life [56], and is a possible protective factor against asthma development [57]. As people with asthma are at an increased risk for mobility problems and musculoskeletal pain [58], interventions focusing on reducing this pain, discomfort and mobility problems are probably essential as well to reduce the time spent sedentary. Not only asthma, but also other chronic lung diseases such as chronic obstructive pulmonary disease (COPD) may induce mobility difficulties, pain, or disability. These factors may predispose an individual to be more socially isolated due to their restrictions in ability to conduct activities of daily living or stigmatized as respiratory symptoms are often associated with tuberculosis in LMICs [59].

For over a decade, targeting increases in moderate to vigorous physical activity has been the emphasis of a large number of exercise training and behavior change interventions in people with COPD [60], but with limited success [61]. The lower levels of physical activity coupled with the often fragile physical and psychological health among patients with COPD may make reducing SB a more suitable conduit for behavior change $[62,63]$. In our study, people with COPD had 1.48 times higher odds for being sedentary eight or more hours. Recent evidence showed as well that SB is an independent predictor of mortality in subjects with COPD, even adjusting for moderate-to-vigorous physical activity and a number of other variables. Mortality was four times higher in subjects with COPD who spend $\geq 8.5 \mathrm{~h} / \mathrm{d}$ in activities requiring $<1.5 \mathrm{MET}$ [64]. Therefore, there is an urgent need to test interventions to reduce SB including the provision of information about the health consequences of SB [65].

Stroke was associated with a 2.0 times higher odds for high SB in our study. A previous qualitative study in Canadian stroke survivors [66] demonstrated that there is also limited awareness of health risks of SB among stroke survivors.

Finally, we also found significant associations between highly sedentary status and hearing problems and visual impairments. Hearing problems [67] and visual impairments [68] should therefore be considered as important risk conditions for SB in LMICs. Stigma and discrimination associated with these chronic conditions and a lack of social support may complicate daily life participation in these populations.

\section{Practical implications and future research}

Our analyses provide further evidence for the need to develop strategies to deal with a sedentary lifestyle in LMICs, and offer important hypotheses for testing in future prospective cohort studies. Our data add to the need to increase awareness among care providers at all levels of care in LMICs of the risks of SB of their patients. Given the evidence in the general population of the benefits of reducing SB, we propose that it is important to test the efficacy and effectiveness of a dual strategy in low resource settings by developing both a smaller group of master trainers/supervisors (e.g. exercise physiologists and physiotherapists) and researchers and a larger group of practitioners (e.g. nurses) who are responsible for minimizing SB in high risk groups. This method has been successfully employed for cognitive behavioral therapy in trials in LMICs [69, 70]. A stepped-care approach, where patients start with selfmanagement in order to reduce their SB, may be a feasible strategy in LMIC settings. Such self-management strategies could, for example, include regular interruptions in sedentary time, such as standing or walking frequently throughout waking hours, during television commercial breaks or while using the phone. Then, if patients do not achieve significant reductions, they could continue with a manualized approach under the supervision of a non-specialist worker (e.g. nurses, occupational therapists). Patients would only be referred to a specialist supervisor (e.g. exercise physiologists and physiotherapists) if no significant reductions occur, for example due to pain, sleep and cognitive problems or mobility problems or associated anxiety and depression. It is known from physical activity research that inclusion of exercise physiologists or physiotherapists reduces dropout rates from lifestyle interventions and consequently improves outcomes [71]. Careful consideration of which strategies would be most efficacious, and evaluation of this stepped-care approach, is essential. The current available evidence is, however, mainly based on evidence from high-income countries and showed that lifestyle interventions focusing on light physical activity participation reduced SB by $24 \mathrm{~min} /$ day $(95 \% \mathrm{CI}=8$ to $41 \mathrm{~min} /$ day, $n=3981)$ while in interventions focusing on reducing time spent sedentary only, SB was reduced by $42 \mathrm{~min} /$ day $(95 \% \mathrm{CI}=5$ to $79 \mathrm{~min} /$ day, $n=62$ ) [72].

Although application of self-monitoring devices and alarms are not easy implementable in low resource settings, a recent International mobile-health Intervention on physical activity, sitting, and weight in adult employees using light-weight, low-cost, non-interactive 
pedometers as a self-monitoring and motivational tool demonstrated reduction in sedentary time. In a 100-day program, participants were encouraged to increase incidental activity such as using stairs and avoidance of sitting. After 100 days, the time spent sedentary reduced in lowincome countries by $0.73 \mathrm{~h}$ per day $(95 \% \mathrm{CI}=-0.75$ to $-0.70 \mathrm{~h}$ ) and by $0.59 \mathrm{~h}$ per day $95 \% \mathrm{CI}=-1.08$ to $-0.10 \mathrm{~h}$ ) in middle-income countries [73].

Efficacy trials of interventions focusing on SB among people with chronic conditions in different cultural settings across LMICs are however urgently needed. If the efficacy and effectiveness of these SB interventions are well established in better equipped scientific settings with trained research staff, the final step will be to fund interventions and initiatives to translate research findings into "real-world" settings while exploring its cost-effectiveness. In order to justify the inclusion of programs focusing on $\mathrm{SB}$ reductions as a routine component in the treatment of chronic diseases and multimorbidity in LMICs, costbenefit analyses should be conducted in order to quantify the financial implications of diverting resources or investing funds into such initiatives. Therefore, next to intervention studies exploring the efficacy of these programs, effectiveness research capable of driving practice change, along with policy-level research, is urgently required. Ministries of health and education will play a critical role in this governance and policy development step. If research shows that reducing SB is efficacious and effective in the prevention and management of chronic diseases and multimorbidity in middle-aged to older adults in LMICs, interventions should be mainstreamed in existing health systems at all levels of care. Finally, since visual impairments and hearing problems are associated with higher levels of SB, future research could explore whether programs providing hearing aids and glasses might assist those in need to become more physically active.

\section{Limitations and strengths}

The current findings should be interpreted in light of some limitations. First, the study is cross-sectional, and cause and effect cannot be deduced. Therefore, it remains unclear whether SB was caused by chronic conditions or vice versa. For example, SB is known to be a risk factor for cardiovascular diseases, while pain caused by arthritis may cause people to be more sedentary. Second, whilst we included all physical health conditions which were assessed within the SAGE, other physical conditions such as tuberculosis, cancer and HIV may have been present and not identified in the study. Therefore, the prevalence of physical multimorbidity is likely to be an underestimation and it is possible that the association between multimorbidity and SB could have differed if data on more chronic physical conditions were available. Third, since the information on chronic conditions and SB was based on self-report, reporting biases may exist. Future research should utilize objective measures of SB. Accelerometers-inclinometers are available that allow for valid and reliable SB behavior. Fourth, by separating the sample into dichotomous SB categories, we were not able to examine how different quantities of SB may affect morbidity. Finally, the present study did not include institutionalized people, which may limit generalizability at a national level. Nonetheless, the strengths of the study include the multi-national scope focused on LMICs, countries which are not investigated yet in the prior research literature. Additionally, we investigated numerous potentially modifiable factors that can be targeted for future interventions aiming to reduce SB.

\section{Conclusions}

The current study demonstrates that middle-aged to older adults with chronic conditions and physical multimorbidity are significantly more sedentary. Future longitudinal research is required to confirm the directionality of our results and explore influential factors. In addition, research on the efficacy and effectiveness of reducing SB in the management of chronic diseases and physical multimorbidity in LMICs should be a priority for funding bodies. Clinicians should consider in particular mobility problems, disability and pain as potential barriers but also other mental health issues such as sleep problems and poor cognition.

\section{Additional file}

Additional file 1: Table S1. Questions used to assess self-reported diagnosis. Table S2. Questions and answer options used for symptomsbased diagnosis. Table S3. Questions used to assess health status. Table S4. Questions and answer options used for endorsement of DSM-IV depression. (DOCX $15 \mathrm{~kb}$ )

\begin{abstract}
Abbreviations
Cl: Confidence interval; HICs: High-income countries; LMICs: Low- and middle-income countries; OR: Odds ratio; SAGE: Study on Global Ageing and Adult Health; SB: Sedentary behavior

\section{Acknowledgements}

None.

\section{Funding}

Brendon Stubbs receives funding from the National Institute for Health Research Collaboration for Leadership in Applied Health Research \& Care Funding scheme. Ai Koyanagi's work is supported by the Miguel Servet contract financed by the CP13/00150 and PI15/00862 projects, integrated into the National R + D + I and funded by the ISCIII - General Branch Evaluation and Promotion of Health Research - and the European Regional Development Fund (ERDF-FEDER). The views expressed in this publication are those of the authors and not necessarily those of the any funding agencies.
\end{abstract}

\section{Availability of data and materials}

The datasets generated during and/or analyzed during the current study are available in the Global Ageing and Adult Health Survey repository, available at http://www.who.int/healthinfo/sage/en 


\section{Authors' contributions}

Access to the Study on Global Ageing and Adult Health Survey data collection was obtained by AK and DV. Analyses were performed by AK and BS. DV wrote a first draft which was reviewed and revised in several rounds by the other co-authors. All authors approved the final version and all authors certify that they have participated sufficiently in the work to believe in its overall validity and to take public responsibility for appropriate portions of its content.

\section{Ethics approval and consent to participate}

Ethical approval was obtained from ethical boards at each study site. Participants give written informed consent. Details are available at http:// www.who.int/healthinfo/sage/en/

\section{Consent for publication}

Not applicable.

\section{Competing interests}

The authors declare that they have no competing interests.

\section{Publisher's Note}

Springer Nature remains neutral with regard to jurisdictional claims in published maps and institutional affiliations.

\section{Author details \\ 'KU Leuven Department of Rehabilitation Sciences, Tervuursevest 101, 3001 Leuven, Belgium. ${ }^{2}$ KU Leuven, University Psychiatric Center KU Leuven, Leuvensesteenweg 517, 3070 Kortenberg, Belgium. ${ }^{3}$ Physiotherapy Department, South London and Maudsley NHS Foundation Trust, Denmark Hill, London SE5 8AZ, UK. ${ }^{4}$ Health Service and Population Research Department, Institute of Psychiatry, Psychology and Neuroscience, King's College London, De Crespigny Park, London Box SE5 8AF, UK. ${ }^{5}$ Faculty of Health, Social Care and Education, Anglia Ruskin University, Chelmsford, UK. ${ }^{6}$ Research and Development Unit, Parc Sanitari Sant Joan de Déu, Universitat de Barcelona, Fundació Sant Joan de Déu, Dr. Antoni Pujadas, 42, Sant Boi de Llobregat, 0883 Barcelona, Spain. ${ }^{7}$ Instituto de Salud Carlos III, Centro de Investigación Biomédica en Red de Salud Mental, CIBERSAM, Monforte de Lemos 3-5 Pabellón 11, 28029 Madrid, Spain.}

Received: 31 July 2017 Accepted: 19 October 2017

Published online: 27 October 2017

\section{References}

1. World Health Organization: World Health Statistics 2016. Monitoring health for the SDGs sustainable development goals. World Health Organization. 2016;

2. Beard HPJR, Bloom DE. Towards a comprehensive public health response to population ageing. Lancet (London, England). 2015;385:658.

3. Fortin M, Stewart M, Poitras M-E, Almirall J, Maddocks H. A systematic review of prevalence studies on multimorbidity: toward a more uniform methodology. The Ann Fam Med. 2012;10:142-51.

4. Picco L, Achilla E, Abdin E, Chong SA, Vaingankar JA, McCrone P, Chua HC, Heng D, Magadi $\mathrm{H}, \mathrm{Ng}$ LL. Economic burden of multimorbidity among older adults: impact on healthcare and societal costs. BMC Health Serv Res. 2016;16:173

5. Vancampfort D, Koyanagi A, Ward PB, Rosenbaum S, Schuch FB, Mugisha J, Richards J, Firth J, Stubbs B. Chronic physical conditions, multimorbidity and physical activity across 46 low-and middle-income countries. Int J Behav Nutr Phys Act. 2017:14:6

6. Fortin M, Lapointe L, Hudon C, Vanasse A, Ntetu AL, Maltais D. Multimorbidity and quality of life in primary care: a systematic review. Health Qual Life Outcomes. 2004:2:1.

7. Lehnert T, Heider D, Leicht H, Heinrich S, Corrieri S, Luppa M, Riedel-Heller S, König $\mathrm{H}-\mathrm{H}$. Review: health care utilization and costs of elderly persons with multiple chronic conditions. Med Care Res Rev. 2011;68:387-420.

8. Gallo JJ, Hwang S, Joo JH, Bogner HR, Morales KH, Bruce ML, Reynolds IIICF. Multimorbidity, depression, and mortality in primary care: randomized clinical trial of an evidence-based depression care management program on mortality risk. J Gen Intern Med. 2016;31:380-6.
9. Abegunde DO, Mathers CD, Adam T, Ortegon M, Strong K. The burden and costs of chronic diseases in low-income and middle-income countries. Lancet. 2007;370:1929-38.

10. Xu X, Mishra GD, Jones M. Mapping the global research landscape and knowledge gaps on multimorbidity: a bibliometric study. Journal of Global Health. 2017;7

11. Autenrieth CS, Kirchberger I, Heier M, Zimmermann A-K, Peters A, Döring A, Thorand B. Physical activity is inversely associated with multimorbidity in elderly men: results from the KORA-age Augsburg study. Prev Med. 2013;57:17-9.

12. Pedersen B, Saltin B. Exercise as medicine-evidence for prescribing exercise as therapy in 26 different chronic diseases. Scand J Med Sci Sports. 2015;25:1-72

13. Bertheussen GF, Romundstad PR, Landmark T, Kaasa S, Dale O, Helbostad JL. Associations between physical activity and physical and mental health-a HUNT 3 study. Med Sci Sports Exerc. 2011;43:1220-8

14. Hupin D, Roche F, Gremeaux V, Chatard J-C, Oriol M, Gaspoz J-M, Barthélémy J-C, Edouard P. Even a low-dose of moderate-to-vigorous physical activity reduces mortality by $22 \%$ in adults aged $\geq 60$ years: a systematic review and meta-analysis. Br J Sports Med. 2015;49:1262-7.

15. Biswas A, PI O, Faulkner GE, Bajaj RR, Silver MA, Mitchell MS, Alter DA. Sedentary time and its association with risk for disease incidence, mortality, and hospitalization in adults: a systematic review and meta-analysis. Ann Intern Med. 2015;162:123-32.

16. Tremblay MS, Aubert S, Barnes JD, Saunders TJ, Carson V, Latimer-Cheung AE, Chastin SF, Altenburg TM, Chinapaw MJ. Sedentary behavior research network (SBRN)-terminology consensus project process and outcome. Int J Behav Nutr Phys Act. 2017;14:75

17. Loyen A, Verloigne M, Van Hecke L, Hendriksen I, Lakerveld J, SteeneJohannessen J, Koster A, Donnelly A, Ekelund U, Deforche B, et al. Variation in population levels of sedentary time in European adults according to cross-European studies: a systematic literature review within DEDIPAC. Int J Behav Nutr Phys Act. 2016;13:71.

18. Ekelund U, Steene-Johannessen J, Brown WJ, Fagerland MW, Owen N, Powell KE, Bauman A, Lee IM. Does physical activity attenuate, or even eliminate, the detrimental association of sitting time with mortality? A harmonised meta-analysis of data from more than 1 million men and women. Lancet. 2016:388:1302-10.

19. Harvey JA, Chastin SF, Skelton DA. How sedentary are older people? A systematic review of the amount of sedentary behavior. J Aging Phys Act. 2015;23:471-87

20. Loprinzi PD. Sedentary behavior and medical multimorbidity. Physiol Behav. 2015;151:395-7.

21. Murray CJ, Barber RM, Foreman KJ, Ozgoren AA, Abd-Allah F, Abera SF, Aboyans V, Abraham JP, Abubakar I, Abu-Raddad LJ. Global, regional, and national disability-adjusted life years (DALYs) for 306 diseases and injuries and healthy life expectancy (HALE) for 188 countries, 1990-2013: quantifying the epidemiological transition. Lancet. 2015;386:2145-91.

22. Forouzanfar $\mathrm{MH}$, Alexander $\mathrm{L}$, Anderson HR, Bachman VF, Biryukov $\mathrm{S}$, Brauer M, Burnett R, Casey D, Coates MM, Cohen A. Global, regional, and national comparative risk assessment of 79 behavioural, environmental and occupational, and metabolic risks or clusters of risks in 188 countries, 1990-2013: a systematic analysis for the global burden of disease study 2013. Lancet. 2015;386:2287-323.

23. Chow CK, Teo KK, Rangarajan S, Islam S, Gupta R, Avezum A, Bahonar A, Chifamba J, Dagenais G, Diaz R. Prevalence, awareness, treatment, and control of hypertension in rural and urban communities in high-, middle-, and low-income countries. JAMA. 2013:310:959-68.

24. Patel V, Araya R, Chatterjee S, Chisholm D, Cohen A, De Silva M, Hosman C, McGuire H, Rojas G, van Ommeren M. Treatment and prevention of mental disorders in low-income and middle-income countries. Lancet. 2007:370:991-1005.

25. Pengpid S, Peltzer K, Kassean HK, Tsala JPT, Sychareun V, Müller-Riemenschneider F. Physical inactivity and associated factors among university students in 23 low-, middle-and high-income countries. International Journal of Public Health. 2015:60:539-49.

26. Prince S, Reed J, McFetridge C, Tremblay M, Reid R. Correlates of sedentary behaviour in adults: a systematic review. Obes Rev. 2017;18(8):915-93.

27. O'Donoghue G, Perchoux C, Mensah K, Lakerveld J, van der Ploeg $H_{\text {, }}$ Bernaards C, Chastin SF, Simon C, O'Gorman D, Nazare J-A. A systematic 
review of correlates of sedentary behaviour in adults aged 18-65 years: a socio-ecological approach. BMC Public Health. 2016;16:163.

28. Kowal P, Chatterji S, Naidoo N, Biritwum R, Fan W, Lopez Ridaura R, Maximova T, Arokiasamy P, Phaswana-Mafuya N, Williams S, et al. Data resource profile: the World Health Organization study on global AGEing and adult health (SAGE). Int J Epidemiol. 2012;41:1639-49.

29. Ekelund U, Steene-Johannessen J, Brown WJ, Fagerland MW, Owen N, Powell KE, Bauman A, Lee I-M, Series LPA, Group LSBW. Does physical activity attenuate, or even eliminate, the detrimental association of sitting time with mortality? A harmonised meta-analysis of data from more than 1 million men and women. Lancet. 2016;388:1302-10.

30. Freeman EE, Roy-Gagnon MH, Samson E, Haddad S, Aubin MJ, Vela C, Zunzunegui MV. The global burden of visual difficulty in low, middle, and high income countries. PLoS One. 2013;8:e63315.

31. Arokiasamy P, Uttamacharya KP, Capistrant BD, Gildner TE, Thiele E, Biritwum RB, Yawson AE, Mensah G, Maximova T, et al. Chronic noncommunicable diseases in 6 low- and middle-income countries: findings from wave 1 of the World Health Organization's study on global Ageing and adult health (SAGE). Am J Epidemiol. 2017;185:414-28.

32. Garin N, Koyanagi A, Chatterji S, Tyrovolas S, Olaya B, Leonardi M, Lara E, Koskinen S, Tobiasz-Adamczyk B, Ayuso-Mateos JL, Haro JM. Global multimorbidity patterns: a cross-sectional, population-based, multi-country study. Journal of Gerontology Series A: Biological Sciences and Medical Sciences. 2016:71:205-14

33. Rose GA. The diagnosis of ischaemic heart pain and intermittent claudication in field surveys. Bull World Health Organ. 1962;27:645-58.

34. Moussavi S, Chatterji S, Verdes E, Tandon A, Patel V, Ustun B. Depression, chronic diseases, and decrements in health: results from the world health surveys. Lancet. 2007;370:851-8

35. Ware J, Jr., Kosinski M, Keller SD: A 12-item short-form health survey: construction of scales and preliminary tests of reliability and validity. Med Care 1996, 34:220-233.

36. Feeny D, Furlong W, Boyle M, Torrance GW. Multi-attribute health status classification systems. Health utilities index. PharmacoEconomics. 1995;7:490-502.

37. Kind P. The Euroqol instrument: an index of health-related quality of life. In: Spiker B, editor. Quality of Life and Pharmacoeconomics in Clinical Trial: Lippincott-raven publishers; 1996. p. 191-201.

38. Stubbs B, Koyanagi A, Schuch FB, Firth J, Rosenbaum S, Veronese N, Solmi M, Mugisha J, Vancampfort D. Physical activity and depression: a large crosssectional, population-based study across 36 low- and middle-income countries. Acta Psychiatr Scand. 2016;134:546-56.

39. Stubbs B, Koyanagi A, Schuch F, Firth J, Rosenbaum S, Gaughran F, Mugisha J, Vancampfort D. Physical activity levels and psychosis: a mediation analysis of factors influencing physical activity target achievement among 204186 people across 46 low- and middle-income countries. Schizophr Bull. 2017 ; 43(3):536-45.

40. Stubbs B, Koyanagi A, Hallgren M, Firth J, Richards J, Schuch F, Rosenbaum S, Mugisha J, Veronese N, Lahti J, Vancampfort D. Physical activity and anxiety: a perspective from the world health survey. J Affect Disord. 2017;208:545-52.

41. Vancampfort D, Koyanagi A, Hallgren M, Probst M, Stubbs B. The relationship between chronic physical conditions, multimorbidity and anxiety in the general population: a global perspective across 42 countries. Gen Hosp Psychiatry. 2017:45:1-6.

42. Kessler RC, Ustun TB. The world mental health (WMH) survey initiative version of the World Health Organization (WHO) composite international diagnostic interview (CIDI). Int J Methods Psychiatric Research. 2004;13:93-121.

43. Koyanagi A, Garin N, Olaya B, Ayuso-Mateos JL, Chatterji S, Leonardi M, Koskinen S, Tobiasz-Adamczyk B, Haro JM. Chronic conditions and sleep problems among adults aged 50 years or over in nine countries: a multicountry study. PLoS One. 2014;9:e114742.

44. Üstün TB, Kostanjsek N, Chatterji S, Rehm J. Measuring health and disability: manual for WHO disability assessment schedule (WHODAS 2.0). In: Geneva; 2010.

45. Tyrovolas S, Koyanagi A, Olaya B, Ayuso-Mateos JL, Miret M, Chatterji S, Tobiasz-Adamczyk B, Koskinen S, Leonardi M, Haro JM. The role of muscle mass and body fat on disability among older adults: a cross-national analysis. Exp Gerontol. 2015;

46. Breen $\mathrm{R}$, Karlson KB, Holm A. Total, direct, and indirect effects in logit and Probit models. Sociol Methods Res. 2013;42:164-91.

47. Stubbs B, Patchay S, Soundy A, Schofield P. The avoidance of activities due to fear of falling contributes to sedentary behavior among community-dwelling older adults with chronic musculoskeletal pain: a multisite observational study. Pain Med. 2014;15:1861-71.

48. Pinheiro MB, Ferreira ML, Refshauge K, Ordoñana JR, Machado GC, Prado LR, Maher CG, Ferreira PH. Symptoms of depression and risk of new episodes of low back pain. A systematic review and meta-analysis. Arthritis Care \& Research. 2015.

49. Hamer M, Stamatakis E. Prospective study of sedentary behavior, risk of depression, and cognitive impairment. Med Sci Sports Exerc. 2014;46:718.

50. Muir SW, Gopaul K, Montero Odasso MM. The role of cognitive impairment in fall risk among older adults: a systematic review and meta-analysis. Age Ageing. 2012;41:299-308

51. Shimada H, Park H, Makizako H, Tsutsumimoto K, Uemura K, Nakakubo S, Hotta $\mathrm{R}$, Suzuki T. Cognitive function and falling among older adults with mild cognitive impairment and slow gait. Geriatr Gerontol Int. 2015;15:1073-8.

52. Tuerk C, Zhang H, Sachdev P, Lord SR, Brodaty H, Wen W, Delbaere K. Regional gray matter volumes are related to concern about falling in older people: a voxel-based morphometric study. Journals of Gerontology Series A: Biomedical Sciences and Medical Sciences. 2015;71:138-44.

53. Singh B, Parsaik AK, Mielke MM, Roberts RO, Scanlon PD, Geda YE, Pankratz VS, Christianson T, Yawn BP, Petersen RC. Chronic obstructive pulmonary disease and association with mild cognitive impairment: the Mayo Clinic study of aging. Mayo Clinical Proceedings. 2013;88:1222-30.

54. Veeranki SP, Downer B, Jupiter D, Wong R. Arthritis and risk of cognitive and functional impairment in older Mexican adults. Journal of Aging and Health. 2017;29:454-73.

55. Fenton SA, van Zanten JJV, Kitas GD, Duda JL, Rouse PC, C-A Y, Metsios GS. Sedentary behaviour is associated with increased long-term cardiovascular risk in patients with rheumatoid arthritis independently of moderate-to-vigorous physical activity. BMC Musculoskelet Disord. 2017;18:131.

56. Mancuso CA, Sayles W, Robbins L, Phillips EG, Ravenell K, Duffy C, Wenderoth S, Charlson ME. Barriers and facilitators to healthy physical activity in asthma patients. J Asthma. 2006;43:137-43.

57. Eijkemans $M$, Mommers $M$, Jos MT, Thijs C, Prins MH. Physical activity and asthma: a systematic review and meta-analysis. PLoS One. 2012;7:e50775.

58. Lunardi AC, Marques da Silva CCB, Rodriques Mendes FA, Marques AP, Stelmach R, Fernandes Carvalho CR. Musculoskeletal dysfunction and pain in adults with asthma. J Asthma. 2011:48:105-10.

59. Van Gemert F, Chavannes N, Nabadda N, Luzige S, Kirenga B, Eggermont C, de Jong $C$, van der Molen T. Impact of chronic respiratory symptoms in a rural area of sub-Saharan Africa: an in-depth qualitative study in the Masindi district of Uganda. Primary Care Respiratory Journal. 2013;22:300-5.

60. Mantoani LC, Rubio N, McKinstry B, MacNee W, Rabinovich RA. Interventions to modify physical activity in patients with COPD: a systematic review. European Respiratory Journal. 2016:ERJ-01744-2015.

61. Cindy Ng LW, Mackney J, Jenkins S, Hill K. Does exercise training change physical activity in people with COPD? A systematic review and meta-analysis. Chronic Respiratory Disease. 2012;9:17-26.

62. Cavalheri V, Straker L, Gucciardi DF, Gardiner PA, Hill K. Changing physical activity and sedentary behaviour in people with COPD. Respirology. 2016;21:419-26.

63. Hill K, Gardiner P, Cavalheri V, Jenkins S, Healy G. Physical activity and sedentary behaviour: applying lessons to chronic obstructive pulmonary disease. Intern Med J. 2015;45:474-82.

64. Furlanetto KC, Donária L, Schneider LP, Lopes JR, Ribeiro M, Fernandes KB, Hernandes NA, Pitta F. Sedentary behavior is an independent predictor of mortality in subjects with COPD. Respir Care. 2017;62:579-87.

65. Cheng SWM, Alison J, Dennis S, Stamatakis E, Spencer L, McNamara R, Sims S, McKeough Z. A behaviour change intervention to reduce sedentary time in people with chronic obstructive pulmonary disease: protocol for a randomised controlled trial. J Phys. 2017;

66. Ezeugwu VE, Garga N, Manns PJ. Reducing sedentary behaviour after stroke: perspectives of ambulatory individuals with stroke. Disabil Rehabil. 2016:1-8.

67. Gispen FE, Chen DS, Genther DJ, Lin FR. Association between hearing impairment and lower levels of physical activity in older adults. J Am Geriatr Soc. 2014;62:1427-33.

68. Marmeleira J, Laranjo L, Marques O, Pereira C. Physical activity patterns in adults who are blind as assessed by accelerometry. Adapt Phys Act Q. 2014; 31:283-96.

69. Naeem F, Saeed S, Irfan M, Kiran T, Mehmood N, Gul M, Munshi T, Ahmad S, Kazmi A, Husain N. Brief culturally adapted CBT for psychosis (CaCBTp): a 
randomized controlled trial from a low income country. Schizophr Res. 2015;164:143-8.

70. Naeem F, Sarhandi I, Gul M, Khalid M, Aslam M, Anbrin A, Saeed S, Noor M, Fatima G, Minhas F. A multicentre randomised controlled trial of a carer supervised culturally adapted CBT (CaCBT) based self-help for depression in Pakistan. J Affect Disord. 2014;156:224-7.

71. Vancampfort DRS, Schuch FB, Ward PB, Probst M, Stubbs B. Prevalence and predictors of treatment dropout from physical activity interventions in schizophrenia: a meta-analysis. Gen Hosp Psychiatry. 2015;39:15-23.

72. Martin A, Fitzsimons C, Jepson R, Saunders DH, van der Ploeg HP, Teixeira PJ, Gray CM, Mutrie N: Interventions with potential to reduce sedentary time in adults: systematic review and meta-analysis. Br J Sports Med 2015: 49(16):1056-1063.

73. Ganesan AN, Louise J, Horsfall M, Bilsborough SA, Hendriks J, McGavigan $A D$, Selvanayagam JB, Chew DP. International mobile-health intervention on physical activity, sitting, and weight: the Stepathlon cardiovascular health study. J Am Coll Cardiol. 2016;67:2453-63.

Submit your next manuscript to BioMed Central and we will help you at every step:

- We accept pre-submission inquiries

- Our selector tool helps you to find the most relevant journal

- We provide round the clock customer support

- Convenient online submission

- Thorough peer review

- Inclusion in PubMed and all major indexing services

- Maximum visibility for your research

Submit your manuscript at www.biomedcentral.com/submit
C) Biomed Central 